\title{
Paralleler Nachweis biologischer Toxine mit einer elektrochemischen Detektionsplattform
}

\author{
Christopher Pöhlmann ${ }^{1}$, Thomas Elßner ${ }^{1}$ und Ralf Wörl ${ }^{2}$ \\ ${ }^{1}$ Bruker Daltonik GmbH, 04318 Leipzig, Permoserstr. 15 \\ ${ }^{2}$ AJ eBiochip, 25524 Itzehoe, Fraunhoferstrasse 1
}

\section{Zusammenfassung}

Der schnelle und zuverlässige Vor-Ort-Nachweis bakterieller Toxine ist von immenser Bedeutung für den Schutz der Bevölkerung vor terroristischen Anschlägen. Das hier vorgestellte elektrochemische Nachweissystem $p T D$ (portable Toxin Detector) erlaubt die parallele und voll-automatische Detektion von bis zu fünf bakteriellen Toxinen innerhalb weniger als 25 Minuten. Der Nachweis basiert auf dem Prinzip eines miniaturisierten elektrochemisch-auslesbaren Sandwich-Immunoassays. Neben Nachweisgrenzen im niedrigen $\mathrm{ng} / \mathrm{mL}-$ Bereich zeichnet sich die entwickelte Methode durch eine spezifische Detektion der einzelnen Analyten aus. Darüber hinaus konnten SEB und Ricin in verschiedenen komplexen Lebensmittelmatrices erfolgreich nachgewiesen werden.

\section{Einleitung}

Die Entwicklung von Multiplex-fähigen, schnellen und sensitiven Detektionsverfahren zum Nachweis von Bakterien, Viren und Toxinen ist essentiell für den Vor-Ort-Nachweis biologischer Gefahrstoffe. Diesem kommt seit dem Auftreten der Anthraxbriefe 2001 und den Ricinbriefen 2003 eine besondere Bedeutung zu. Eine zeitnahe Einleitung geeigneter medizinischer Gegenmaßnahmen ist nur mit einem zuverlässigen VorOrt-Nachweissystem möglich. Bakterielle Toxine wie die Botulinum Neurotoxine (Serotypen BoNT/A BoNT/G) und Staphylokokken Enterotoxine (SEA - SEU) sowie pflanzliche Toxine wie Ricin und Abrin besitzen ein erhebliches Potential als biologische Kampfstoffe in Erscheinung zu treten, da sie leicht verfügbar sind, eine hohe Toxizität aufweisen und relativ einfach auszubringen sind [1].

Da Toxine auch in Abwesenheit des produzierenden Organismus toxisch sind, basiert ihr Nachweis schwerpunktmäßig auf der Detektion des Proteins mittels Immunoassay-basierten Methoden oder mittels spektrometrischer Verfahren. Elektrochemische Nachweisverfahren haben gegenüber konventionellen Methoden den Vorteil, dass sie hochsensitiv und schnell auslesbar sind. Daneben zeigen sie Potential zur Miniaturisierung und Automatisierung und somit zur Konstruktion von tragbaren Nachweistechnologien [2].

\section{Experimenteller Teil}

\subsection{Materialien}

Für die Experimente wurden verschiedene BoNT-Serotypen (Metabiologics Inc., Madison, USA), SEA, SEB (Sigma-Aldrich, Steinheim) und Ricin (Toxin Technology Inc., Sarasota, USA) verwendet.

Für die Toxindetektion wurde das pTD inklusive pTD Control Software zusammen mit dem pTD Toxin Test Kit BWA I und dem pTD Washing Kit (Bruker Daltonik, Leipzig) verwendet. Die Probenvorbereitung verschiedener Probenmatrices wurde mit Hilfe des pTD Sample Preparation Kit (Bruker Daltonik, Leipzig) durchgeführt.

\subsection{Detektion von Toxinen in Probenmatrices}

Der Nachweis von Toxinen in Probenmatrices (verschiedene flüssige und feste Proben) erfolgte nach Herstellerangaben mit dem pTD Sample Preparation Kit und dem pTD Toxin Test Kit BWA I (Bruker 
Daltonik, Leipzig). $1 \mathrm{~mL}$ flüssige Probe, z.B. Cola, Wasser oder Milch, wurden mit $1 \mathrm{~mL}$ Probenpuffer vermischt. Die Probensuspension wurde anschließend mit einer finalen Konzentration von $2 \mathrm{ng} / \mathrm{mL}$ SEB versetzt und für $2 \mathrm{~h}$ bei Raumtemperatur inkubiert. Daraufhin wurde die Probensuspension durch einen Spritzenvorsatzfilter filtriert, um mögliche partikuläre Substanzen zu entfernen. 0,5-1,0 mL Probe wurden für die Messung mit dem pTD verwendet. Im Falle fester Proben wurden etwa 0,5-1,0 g Probe mit $5 \mathrm{~mL}$ Probenpuffer vermischt und mit einer finalen Konzentration von $2 \mathrm{ng} / \mathrm{mL}$ SEB versetzt. Die weiteren Probenvorbereitungsschritte entsprachen denen für flüssige Proben.

\section{Ergebnisse und Diskussion}

Der Toxinnachweis mittels pTD-Plattform (Bild 1 A) basiert auf dem Prinzip eines miniaturisierten Sandwich-Immunoassays: Toxin-spezifische Fängerantikörper werden auf einem elektrochemischen Biochip immobilisiert (Bild 1 B). Die toxinhaltige Probe sowie die für die Detektion erforderlichen Reagenzien werden nacheinander in einem automatisierten Verfahren in der pTD-Plattform auf dem Chip inkubiert. Der Nachweis erfolgt nach etwa 25 Minuten durch die enzymatische Umsetzung eines Substrats, wodurch ein spezifisches elektrochemisches Signal die Anwesenheit eines Toxins signalisiert (Bild 1 C).

Der etablierte Toxin-Chip erlaubt die parallele Detektion von BoNT/A, /B, /E, SEB und Ricin. Weiterhin weist jeder Chip interne Positiv- und Negativkontrollen zur Validierung und Quantifizierung auf (Bild 1 B). Alle Messsignale werden in Bezug zur Positivkontrolle gesetzt, die als 100\% definiert wird. Sowohl die Durchführung als auch die Auswertung einer Messung erfolgt automatisch mit einer Wizard-ähnlichen Software.

(A)

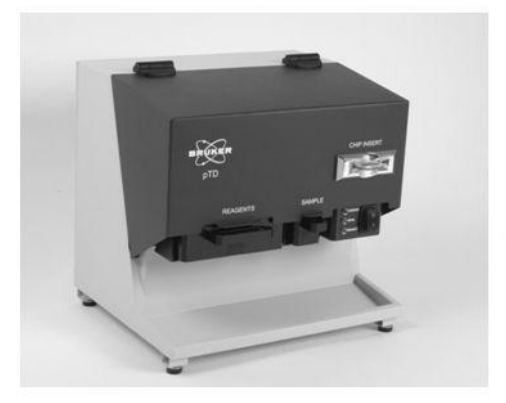

(B)

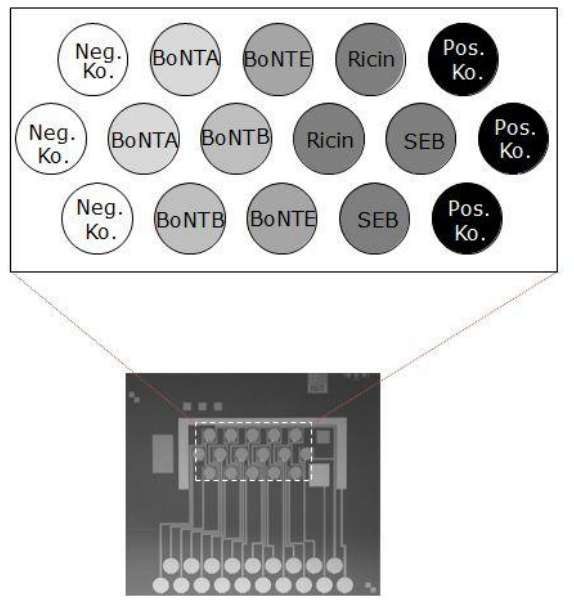

(C)

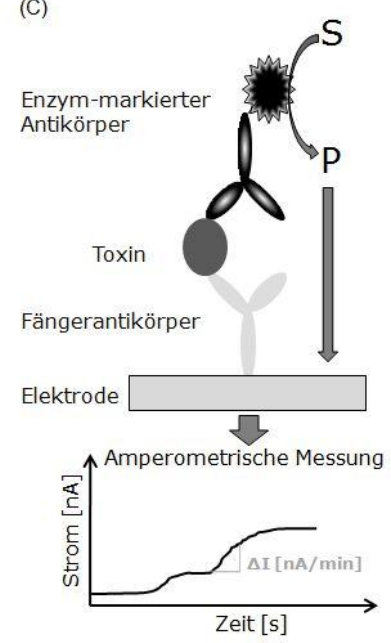

Bild 1 Nachweis von biologischen Toxinen mittels pTD-System. (A) Der elektrochemische Detektor pTD mit Reagenzien- und Probenhalter bestückt. (B) Darstellung der Belegung der 16 Elektrodenpositionen des Toxin-Chips. (C) Prinzip der Detektion von Toxinen mittels Sandwich-Immunoassay.

\subsection{Sensitivität und Nachweisgrenzen der elektrochemischen Toxindetektion}

Aufgrund der hohen Toxizität biologischer Toxine (z. B. $\mathrm{LD}_{50}$ von BoNT/A ist $1 \mathrm{ng}$ pro kg Körpergewicht oder $\mathrm{LD}_{50}$ von SEB ist 0,02 - $10 \mu \mathrm{g}$ pro kg Körpergewicht [1]) erfordern zuverlässige Nachweismethoden eine außergewöhnliche Empfindlichkeit, um sinnvolle Aussagen über die Anwesenheit bedrohlicher Mengen an Toxinen zu treffen.

Zur Bestimmung der Nachweisgrenze des hier vorgestellten elektrochemischen Nachweissystems wurden unterschiedliche Konzentrationen an Toxinen für die Messung mit Toxin-Chips eingesetzt und mit den erhaltenen normalisierten Messsignalen korreliert. Man erhält unabhängig vom verwendeten Analyten eine sigmoidale Kalibrationskurve wie in Bild 2 exemplarisch für SEB dargestellt.

Analog wurden die Nachweisgrenzen für alle anderen Toxine bestimmt (Tafel 1). Die Nachweisgrenze ist definiert als der niedrigste Messwert über dem Mittelwert des Blindwertes plus dreimal dessen Standardabweichung. 
Die ermittelten Nachweisgrenzen im niedrigen ng/mL-Bereich erlauben den zuverlässigen Nachweis dieser biologischen Toxine innerhalb weniger als 25 Minuten in einem automatisierten Prozess. Somit können nach Messung mit dem pTD schnell Aussagen bezüglich Anwesenheit gefährlicher Konzentrationen an Toxinen etwa in Lebensmitteln getroffen werden.

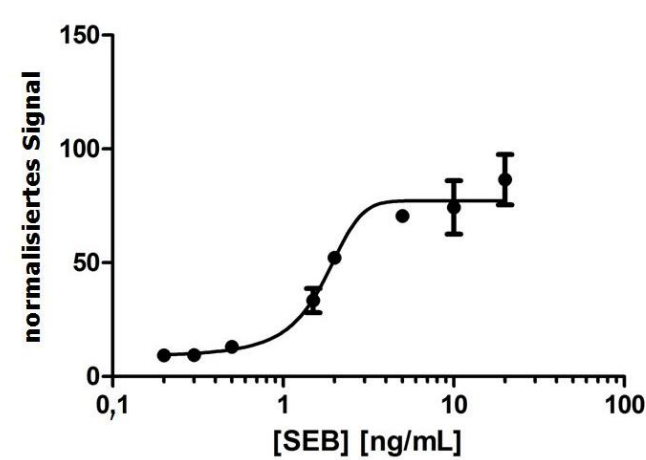

Bild 2 Sensitivität des elektrochemischen SEB-Nachweises. Auftragung unterschiedlicher SEB-Konzentrationen gegen die normalisierten Signale.

Tafel 1 Ermittelte Nachweisgrenzen der elektrochemischen Toxindetektion ${ }^{1}$

\begin{tabular}{l|l}
\hline Toxin & Nachweisgrenze \\
\hline BoNT/A & $8 \mathrm{ng} / \mathrm{mL}$ \\
\hline BoNT/B & $5 \mathrm{ng} / \mathrm{mL}$ \\
\hline BoNT/E & $150 \mathrm{ng} / \mathrm{mL}$ \\
\hline SEB & $0,3 \mathrm{ng} / \mathrm{mL}$ \\
\hline Ricin & $0,5 \mathrm{ng} / \mathrm{mL}$
\end{tabular}

${ }^{1)}$ Die angegebenen Nachweisgrenzen sind definiert als der niedrigste Messwert über dem Mittelwert des Blindwertes plus dreimal dessen Standardabweichung.

\subsection{Selektivität der Toxindetektion}

Neben der Sensitivität ist die Selektivität der entscheidende Parameter für den Einsatz von Biochips in der diagnostischen Mikrobiologie. Nach Applikation von BoNT/A, BoNT/B, BoNT/E, Ricin oder SEB zur Messung mit dem Toxin-Chip ergab sich für jeden einzelnen Analyten ein selektives Signal auf den mit spezifischen Fängerantikörpern besetzten Elektrodenpositionen (Bild 3). Dagegen zeigten die Negativkontrollelektroden bzw. Elektroden mit unspezifischen Fängerantikörpern kein signifikantes Messsignal.

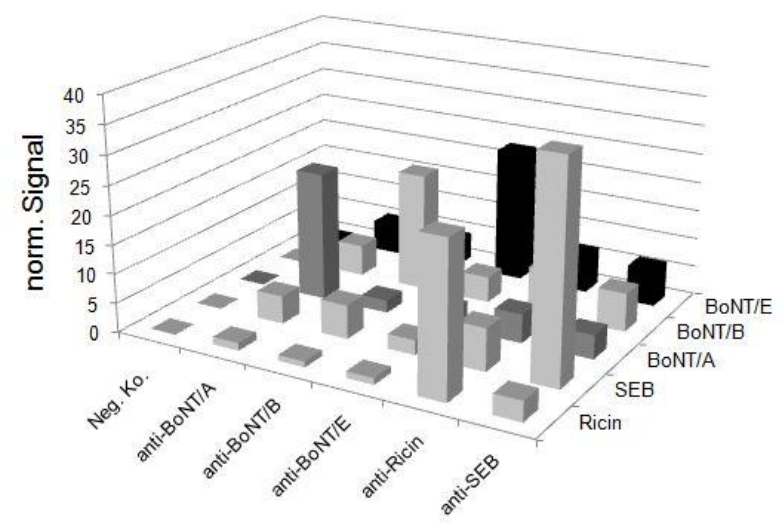

Fängerantikörper

Bild 3 Selektivität des Toxinnachweises mit dem pTD. Nachweis von $2-500 \mathrm{ng} / \mathrm{mL}$ Toxin mittels Toxin-Chip und pTD. 
Nah verwandte Moleküle wie Abrin und andere Lektine bzw. andere BoNT- oder SE-Typen werden nicht detektiert.

Um eine sichere Aussage über eine Gefahrenlage zu treffen, muss eine diagnostische Methode in der Lage sein, spezifisch einen Analyten in der Gegenwart eines Überschusses an verwandten Molekülen zu identifizieren. Mit Hilfe des pTD konnte gezeigt werden, dass SEB auch bei einem 1000-fachen Überschuss an nah-verwandten SEA an der Nachweisgrenze detektierbar ist. Dagegen wird in Gegenwart von $30 \mathrm{ng} / \mathrm{mL}$ SEA kein signifikantes Signal generiert.

\subsection{Nachweis von Toxinen in komplexen Matrices}

Sowohl BoNTs als auch SEB wurden als Ursachen von Lebensmittelvergiftungen identifiziert. Ein mögliches Szenario eines bioterroristischen Anschlags zielt auf die Kontamination von Lebensmitteln und Trinkwasser ab. Deshalb wurde untersucht, inwieweit die pTD-Plattform in der Lage ist, Toxine in unterschiedlichen Probenmatrices zuverlässig zu detektieren. Jede untersuchte Probematrix wurde dazu mit 2 $\mathrm{ng} / \mathrm{mL}$ SEB versetzt (Bild 4). Als Positivkontrolle wurde $2 \mathrm{ng} / \mathrm{mL}$ SEB in Probenpuffer verwendet, während als Negativkontrolle Puffer ohne SEB getestet wurde. SEB konnte in allen untersuchten Probenmatrices mit dem pTD nachgewiesen werden. Vor allem die Matrices Apfelsaft, Honig und Teepulver führten jedoch zu einem deutlichen Abfall der Signalstärke. Offensichtlich beeinflussen Matrixeffekte deutlich die AntikörperAntigen-Bindung, wahrscheinlich durch Maskierung von Oberflächen-exponierten Epitopen.

(A)

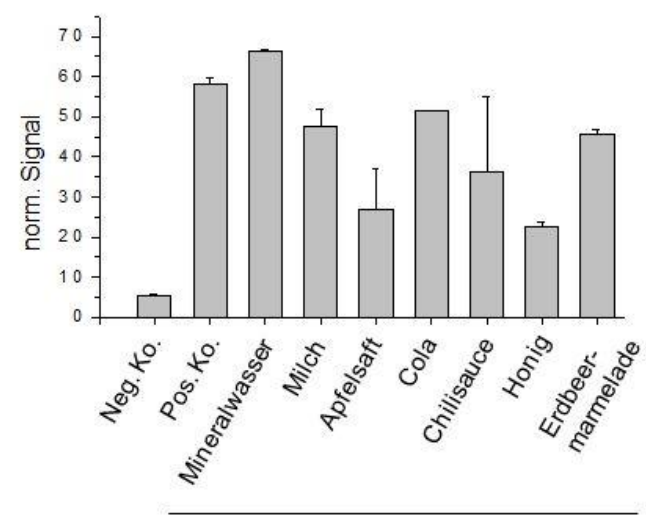

$2 \mathrm{ng} / \mathrm{mL}$ SEB in
(B)

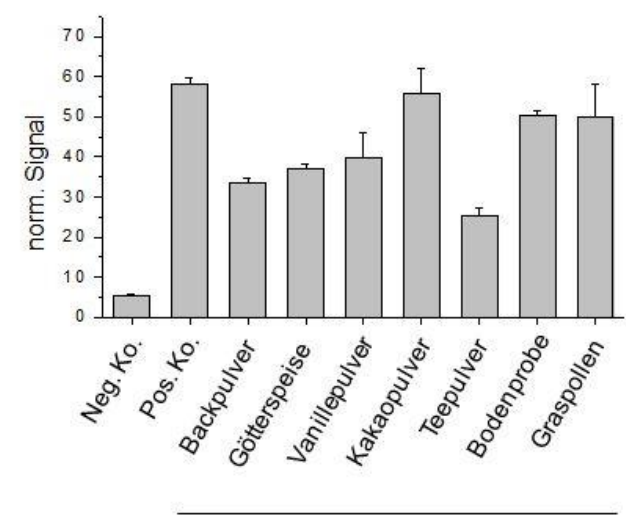

$2 \mathrm{ng} / \mathrm{mL}$ SEB in

Bild 4 Nachweis von SEB in unterschiedlichen Probenmatrices. (A) Detektion von $2 \mathrm{ng} / \mathrm{mL}$ SEB in flüssigen und semi-festen Probenmatrices. Als Negativkontrolle (Neg. Ko.) wurde Probenpuffer ohne Toxin verwendet, während als Positivkontrolle (Pos. Ko.) 2 ng/mL SEB in Probenpuffer verwendet wurde. (B) Nachweis von 2 ng/mL SEB in festen Probenmatrices. Dabei wurden Negativ- und Positivkontrolle wie in (A) verwendet.

Weiterhin wurde der Nachweis verschiedener nicht-aufgereinigter Toxine (z. B. Nachweis von SEB aus dem Kulturüberstand verschiedener SE-produzierender Staphylococcus aureus-Stämme, Ricin isoliert aus unterschiedlichen Ricinus communis-Samen, künstlich mit Ricin kontaminierte Lebensmittelmatrices) ohne langwierige und komplexe Probenvorbereitungsschritte demonstriert.

\section{Literatur}

[1] J.C. Burnett, E.A. Henchal, A.L. Schmaljohn, S. Bavari: The evolving field of biodefence: therapeutic developments and diagnostics. Nat Rev Drug Discov. 4, S. 281-297, 2005.

[2] J. Wang: Electrochemical biosensors: Towards point-of-care cancer diagnostics. Biosens. Bioelectron. 21, S. 1887-1829, 2006. 